\title{
Investigation of temperature distribution during plastic deformation of stainless steel
}

\author{
by S.P. Gadaj, W.K. Nowacki and E.A. Pieczyska
}

Center of Mechanics, Institute of Fundamental Technological Research, CEwietokrzyska street 21, 00-049 Warsaw, Poland

\section{Abstract}

The object of the work was to find the temperature fields and their evolution during tensile test and simple shear test of metal in order to use them in the examination of material's behaviour.

In initial stage of metal's elongation, the temperature distribution allows to determine the beginning of plastic deformation. In the homogeneous state of stress temperature measurements enable to investigate a process of energy storage during the plastic deformation. Heterogeneous temperature distribution observed in the subsequent, advanced state of deformation are related to evolution of the localized plastic deformation, leading to necking and damage.

Furthermore, examination of the temperature distribution obtained during static simple shear test enables us to confirm the occurrence of macroscopic shear bands, calculated in theoretical approach.

\section{Introduction}

As we know from experiments, heating of a body produces changes in stress, strain and temperature. Conversely, deformation of a body is associated with a change of its internal energy, and consequently in its temperature distribution. This mutual interaction between deformation and the temperature fields is called thermomechanical coupling. Effects of thermomechanical coupling, occurring in nature, have a long history both in theoretical and experimental approaches.

Considerable increase in capabilities and in accuracy of temperature measurement, related to elastic and plastic deformation of metals, appearing after the non-contact measurement approaches and based on detection of infrared radiation, were introduced [1-3]. This type of measurement system was used in the present investigations. It consists of a thermovision camera, coupled to a computer system of data acquisition and conversion, which enables to store the obtained temperature distributions on the hard disc of a computer and to further process it by dedicated software. The accuracy of temperature measurement is about $0.2 \mathrm{~K}$; in some approaches it can be higher, even $0.01 \mathrm{~K}$.

All the tests were performed in a Instron testing machine, most of them during a tensile test, on flat samples of austenitic steel. In order to secure higher and more homogeneous emissivity, the surface of the samples was blackened by a carbon powder.

\section{Identification of the beginning of plastic deformation based on temperature measurements.}

Empirical identification of the boundary between the elastic and the plastic regimes of deformation is complex and ambiguous, since ambiguous is also the definition of the yield point as well as the term used for elastic deformation. The methods utilizing the thermal emission are based on a qualitative change of the temperature behaviour of specimen under mechanical loading. During elastic deformation, these changes are, in general, linearly dependent on stress and are negative in the case of the elongation test. Otherwise, during the plastic deformation, always accompanied by an increase in temperature, the temperature changes are nonlinear. The change of temperature $\Delta T$ of a specimen, subjected to adiabatic uniaxial elastic deformation, can be described as follows:

$$
\Delta T=-\frac{\alpha T \Delta \sigma_{s}}{c_{\sigma}},
$$


where: $\alpha$ - coefficient of linear thermal expansion, $T$ - absolute initial temperature,

$$
\Delta \sigma_{s}-\text { isentropic change in stress, } c_{\sigma} \text {-specific heat capacity. }
$$

Assuming that the material coefficients used in Eq. 1 are constant, a linear dependence between the change of temperature and the stress describes the elastic deformation $[3,4]$ :

$$
\Delta T_{e l}=-k \Delta \sigma_{s},
$$

Using this theory, the investigation of the beginning of plastic deformation of stainless steel was carried out. The specimen was subjected to several, subsequent tensile tests. It means, that after deformation to $1 \mathrm{~mm}$ (beyond the yield point), the specimen was unloaded, cooled in ambient temperature and strained to $1 \mathrm{~mm}$ again. By operating in this way, five such cycles were performed. The loading vs. elongation, the loading and elongation vs. time and the distribution of infrared radiation were continously registered.

Change in temperature of the specimen subjected to the first cycle of deformation, plotted vs. stress, is shown in Figure 1. In the initial stage of elongation that change is linearly dependent on stress. After this smooth, quasi-reversible decrease in temperature, called thermoelastic effect, the sample heats up due do initiation and evolution of the mechanisms of plastic deformation, involving energy dissipation.

This means, that the point $P$ of departure of the straight line $\Delta T=-k \sigma_{s}$, describing the elastic deformation, from the plot of measured temperature of the specimen, indicates the beginning of plastic deformation [4]. Stress value corresponding to point $P$ is the true macroscopic flow stress. This value is lower than $\sigma_{g}$, obtained as the elastic limit on the basis of the stress - strain curve.

During the subsequent tensile tests (Figure 2), the measured temperature decrease was each time larger. Moreover, the points indicating the beginning of plastic deformation are moving in the direction of higher values of stresses (Figure 3). This may be caused by the fact, that the material of the specimen, hardened in the previous cycles of deformation, can deform elastically longer. It is because that the easy mechanisms of plastic deformation, active in the first (previous) loading test, were used. The deforming material is able each time to absorb more elastic energy without fundamental change of its structure.

\section{Stored energy measurement}

A metal subjected to stress deforms either elastically or plastically. The plastic deformation permanently affects the internal energy of a body. This change, named the stored (latent) energy, remains in the structure of the metal. The effect of energy storage is explained by the occurrence of elastic forces between grains or blocks of similarly oriented grains [4] and, mostly, by generation, motion, blocking, and annihilation of microstructure defects, mainly dislocations, leading to changes in their density and arrangements [3-6]. The amount of the energy experimentally found depends on the applied method of its measurement [3]. The method elaborated by our team and used in our investigations is a modification of the, so-called, singlestep method of the stored energy measurement, based on a direct application of the first law of thermodynamics $[1,2,5,6]$. Energy stored $\left(E_{s}\right)$ is obtained here as the difference between the plastic work done on a body $\left(E_{w}\right)$ and the evolved heat $(Q)$ :

$$
E_{s}=E_{w}-Q
$$

It is easy to find $E_{w}$. In case of a tensile test it is determined from the force vs. elongation plot $F(\Delta l)$, obtained during the experiment (Figure 4):

$$
E_{w}=\int_{0}^{\Delta l} F(\Delta l)-E_{e l}
$$

where: $E_{e l}$ - energy spent during elastic deformation. 
The problem is to find the evolved $Q$. The infrared distribution, registered by the thermovision equipment, allows us to obtain the temperature evolution of the sample subjected to deformation (Figure 4).

In our approach the heat $Q$ was obtained by simulation of the process, in which the supplied energy is totally converted into heat. In this order we use the electrical power. The idea was to deliver such an amount of the electrical energy to the unstrained sample, that the temperature increase during the simulation was the same as that during the deformation. Knowing the parameters of electrical power it is possible to find $E_{s}$. Finally:

$$
E_{s}=E_{w}-\int_{0}^{t} P(t) d t+Q_{i n},
$$

where: $P(t)$ - electrical power supplied to the specimen, $Q_{i n}$ - heat needed for overcoming the thermoelastic effect.

The described method allows us to evaluate $E_{s}$ continuously, without interruption of the process; and it is the main, very important advantage of this method. Hence, this method can be applied only to the homogeneous stage of deformation.

Looking at the obtained results, it is seen, that the energy stored is an increasing function of deformation (expended energy), but the rate of this increase is lower after a certain stage of deformation (Figure 5). Continuity of the energy storage measurements made according to this method allows to calculate a dependence $\frac{d E_{s}}{d E_{w}}=f\left(E_{w}\right)$ and gives information about the energy conversion at each instant of the process of deformation [5]. On the other hand, the energy stored presented as a function of second power of stress is represented by a straight line (Figure 6):

$$
E_{s}=k \times \sigma^{2},
$$

where $k$ - coefficient depending on the used material. Moreover, comparing the dependence $E_{s}\left(\sigma^{2}\right)$ obtained for the same material prepared in a different manner (Fig. 6 ), it has been found that:

$$
E_{s}=E_{0}+k \sigma^{2}
$$

where $E_{0}$ is an estimation of the value of energy supplied to the specimen during initial treatment [6].

\section{Asymmetry of temperature distribution on surface of specimens subjected to static simple shear}

Papers concerning simple shear tests, with an experimental approach, are limited to analysis of mechanical curves, texture and microscopic pictures obtained for such a deformed material, while theoretical approaches concern analysis of the shear testing under isothermic, or adiabatic conditions.

The present investigation of static simple shear were carried out in real, nonadiabatic conditions. Their goal was to obtain both the mechanical curves and the temperature changes of the shear areas of specimens. A specially designed grip was used to transform the compression to the simple plane state of shear. The thin sheet specimen of stainless steel were placed in this grip fitted in the testing machine acting in compression.

The process of shear, like any other plastic deformation, is accompanied by an increase of temperature (Fig. 7B). At deformation higher than $70 \%$, the mechanical curve $\sigma(\gamma)$ ceases to be smooth, that proves that the mechanisms of deformation (Fig. 7A) have changed. It follows from theoretical consideration [7], that during the simple shear test at deformation higher than $\approx 70 \%$, localisation of plastic deformation, called the macroscopic shear band, occurs. The macroscopic 
thear band develops at a certain angle to the shear path. Suitable processing of thermal pictures, obtained during the shear test, allowed to confirm the validity of this model. Taking advantage of used computer system, nine segments intersecting the shear path perpendicularly to the shear dlrection, were chosen on the thermograms obtained in the non-homogeneous range of deformation (Fig. coloured). The distribution of temperature, determined along these segments, lumed out to show asymmetry, related to the macroscopic shear band. Approximation of these distributions by mathematical functions, allowed to find and to investigate the localized plastic deformation, predicted by the theoretical approach.

\section{Conclusion}

As a conclusion one should to notice that, in spite of the fact that other possibilities of Investigation of the elastic and piastic deformation exist, the present one, based on the contactless temperature measurements related to mechanical characteristics, enables to monitor the evolution of plastic deformation in metals during straining.

\section{REFERENCES}

[1] GADAJ (S.P.), GAEKOWSKA-PIECZYSKA (E.), KACZMAREK (J.), OLIFERUK (W.), Modified Method for Determination of Energy Stored in Metal During Its Deformation, Bull. Acad. Polon. Sci.,Seria Sci. Techn., v.XXX, 1-2, 1982.

[2] OLIFERUK (W.), GADAJ (S.P.), GRABSKI (MW.), Energy Storage during the Tensile Deformation of Armco Iron and Austenitic Steel, Mater. Sci. Eng., 70, 1985, 131.

[3] BEVER (M.B.), HOLT (D.L.), TITCHENER (A.L.), The Stored Energy of Cold Work, Progress in Materials Science, 25, 1973.

[4] PIECZYSKA (E.), Critical Point of the Elastoplastic Transition in ternis of Thermomechanical Coupling, J. of Appl. Mech., 1996, p. 281-306.

[5] OLIFERUK (W.), SWIATNICKI (W.A.), GRABSKI (MW.), Rate of Energy Storage and Microstructure Evolution during the Tensile Deformation of Austenitic Steel, Mater. Sci. Eng., 1993, p. 55-63.

[6] GADAJ (S.P.), The Investigation of Energy Storage in Metal's Surface Layer afier Its Machinning, Ph.D.Thesis, Warsaw, 1991.

[7] GADAJ (S.P.), NOWACKI (W.K.), PIECZYSKA (E.A), Changes of temperature during the simple shear test of stainless steel, Arch. Mech., 48, 4, 1996 p. 779-788 


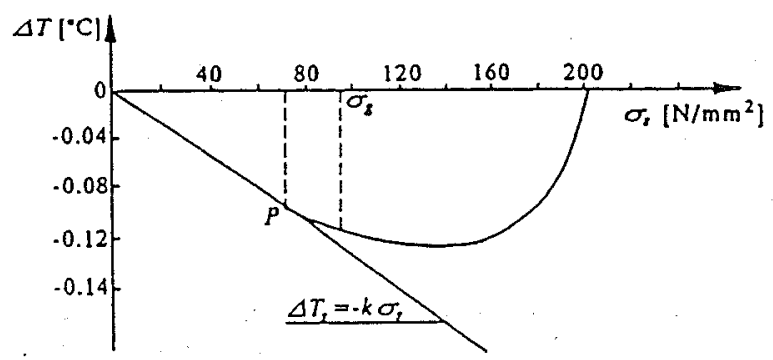

Figure 1. Temperature change vs. stress for the sample of stainless steel
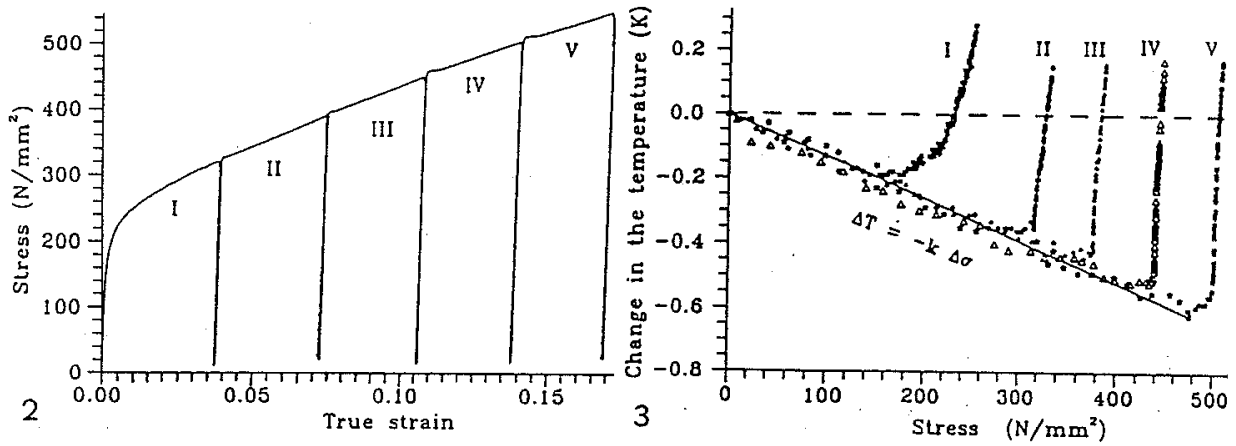

Figures 2,3. Loading vs. elongation (2) and changes of temperature vs stress (3) of specimens of stainless steel during subsequent cycles of deformation

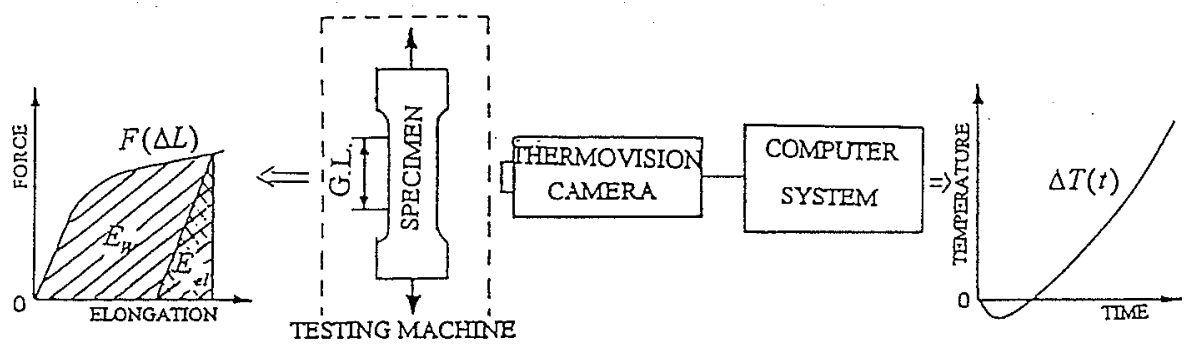

Figure 4. The idea of determining the energy storage during tensile test 


\section{http://dx.doi.org/10.21611/qirt.1996.049}
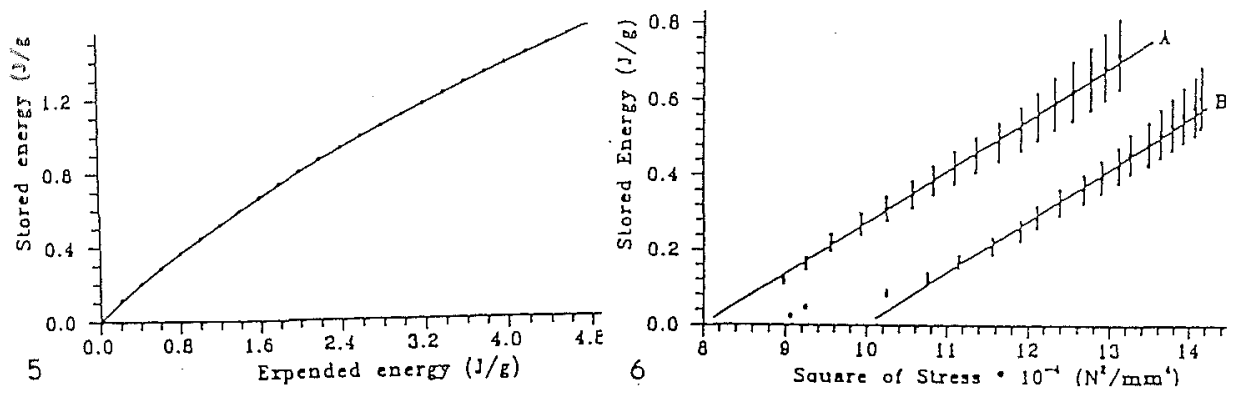

Figures 5,6. Stored energy $E_{s}$ vs. expended energy $E_{w}$ of stainiess steel during tension (5), and stored energy $E_{s}$ vs. square of stress, $\sigma^{2}$ for the sampless of stainless steel (6):

A - after full annealing, B - after burnishing

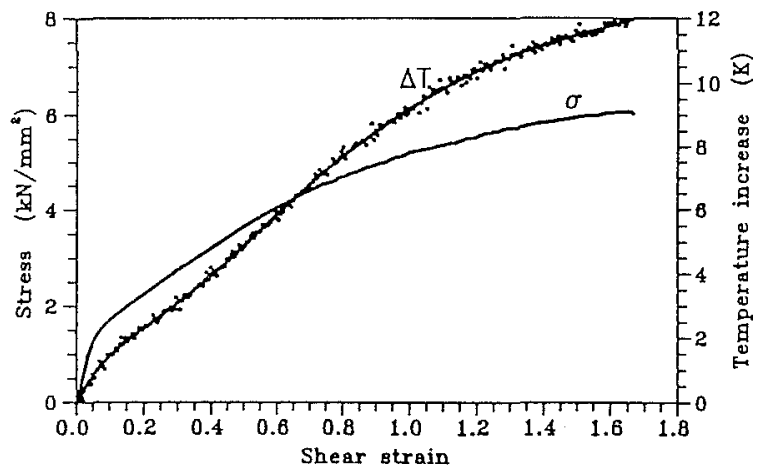

Figure 7. Dependences of: A - shear stress $\sigma$ and B - temperature change $\Delta T$ vs. shear strain $\gamma$ of specimen of stainless steel subjected to shear test 